\title{
Deformation of crosslinked semiflexible polymer networks
}

\author{
David A. Head ${ }^{1,2}$, Alex J. Levine ${ }^{2,3}$, and F.C. MacKintosh ${ }^{1,2}$ \\ ${ }^{1}$ Division of Physics \&5 Astronomy, Vrije Universiteit $1081 \mathrm{HV}$ Amsterdam, The Netherlands \\ ${ }^{2}$ The Kavli Institute for Theoretical Physics, University of California, Santa Barbara CA 93106, USA and \\ ${ }^{3}$ Department of Physics, University of Massachusetts, Amherst MA 01060, USA
}

(Dated: October 25, 2018)

\begin{abstract}
Networks of filamentous proteins play a crucial role in cell mechanics. These cytoskeletal networks, together with various crosslinking and other associated proteins largely determine the (visco)elastic response of cells. In this letter we study a model system of crosslinked, stiff filaments in order to explore the connection between the microstructure under strain and the macroscopic response of cytoskeletal networks. We find two distinct regimes as a function primarily of crosslink density and filament rigidity: one characterized by affine deformation and one by non-affine deformation. We characterize the crossover between these two.
\end{abstract}

PACS numbers: 87.16.Ka, 62.20.Dc, 82.35.Pq

The study of biopolymer networks and gels lies at the heart of the understanding of the mechanical properties of the cytoplasm since the mechanical rigidity of the intracellular material is largely governed by the cytoskeleton, a complex network of filamentous proteins, cross links, and other associated proteins [1]. A key player in this cytoskeleton is F-actin, which exhibits significant rigidity on the cellular scale. The material properties of such semi-flexible polymer networks also pose complex and current problems in polymer physics. Many of the most basic questions concerning these common and important networks, such as how they deform under stress, remain unanswered. The bending rigidity of such polymers introduces a new microscopic elastic parameter that can have consequences for the macroscopic elastic coefficients of the bulk, semi-flexible gel. This changes the traditional rubber elasticity model for the bulk properties of gels composed of cross-linked flexible polymers.

In this letter, we examine a simple model for crosslinked rods that not only allows us to quantitatively test the relationship between the microscopic and macroscopic elastic coefficients of a randomly cross-linked network, but also sheds light on the intimately related issue of the spatial distribution of the network strain. Among the most fundamental properties of polymer networks is the way in which they deform under stress. Since the classical theories of rubber elasticity [2] it has been suggested that this deformation is affine, i.e., that the strain is uniform, as for a sheared Newtonian fluid at low Reynolds number. While this leads to relatively good agreement with experiments for rubber-like networks, much evidence and continuing efforts concern systematic deviations that point to non-affine network strains at a microscopic scale.

The assumption that the deformation field is affine down to length scales comparable to the smallest microscopic scales in the material is a great simplification that allows one to construct quantitative theories relating the macroscopic elastic constants of a gel to the microscopic properties of its constituent polymers. The validity of the affine approximation for this class of semiflexible polymer materials has, however, been the subject of some debate [3, 4, 5]. Whether the deformation field is affine or not depends, of course, on length scale; clearly at the scale of the entire sample, all deformations are trivially affine when subject to simple shear. We explore whether this self-averaging property of the deformation field extends to shorter length scales for semiflexible networks. We show that the degree of non-affine strain is a function of length scale and degree of crosslinking. Specifically, we find that the range of non-affine strain can extend well beyond the mesh size, or correlation length, the typical separation between filaments [6]. This occurs near the point of rigidity percolation for the network, or for highly flexible filaments. This results in elastic moduli governed primarily by bending of filaments under non-affine strain, consistent with [4, 5]. In contrast, we find that these networks become increasingly affine, even down to the smallest scales of the network, e.g. the mesh size, at high cross link density, high molecular weight, or for rigid filaments. Here, we find that the bulk elastic moduli converge to those predicted from affine theory [3]. We also quantify the degree of non-affine strain and show that this is, indeed, dependent on the length scale.

As we focus on the zero-frequency, or static properties of the system we may ignore the complexities of the network-solvent interaction. To isolate the importance of semi-flexibility upon network properties, we also ignore the complex, nonlinear response of the individual F-actin filaments $[3,4,7]$. We instead study in detail the dependence of the bulk shear modulus and Young's modulus of the material upon the cross-link density of the polymer gel as well as the bending and extension moduli of the individual filaments. We model the network filaments via the Hamilitonian per unit length $(\delta s)$ for a filament

$$
\frac{\delta \mathcal{H}}{\delta s}=\frac{\mu}{2}\left(\frac{\delta l}{\delta s}\right)^{2}+\frac{\kappa}{2}\left(\frac{\delta \theta}{\delta s}\right)^{2}
$$


The first term takes into account the extensional deformation of the filament $\delta l(s)$ as a function of arc-length, $s$ with modulus $\mu$. The second term determines the energy stored in the filament due to bending: the local tangent of the filament makes an angle $\theta(s)$ with respect to the $\hat{x}$ axis and the bending modulus of the filament is $\kappa$. Note that both of these terms are quadratic. We do not explore nonlinearities, such as buckling when compressed beyond the Euler instability, at the scale of individual filaments. While such nonlinear effects are expected at increasing strains and finite temperature [3], we seek to understand here the fundamental properties of semi-flexible networks and thus address only the linearized version of the problem. We note, however, that thermal fluctuations can result in an effective modulus $\mu \sim \kappa^{2} /\left(k T l^{3}\right)$ for a segment of length $l$. Thus, we consider $\mu$ and $\kappa$ to be independent parameters, even though they are both determined by single filament elastic properties and geometry at $T=0$ [8]. Clearly the full exploration of these networks at finite temperature presents an interesting challenge; understanding the $T=0$ mechanical properties of these networks, however, is the requisite first step towards this more ambitious program. In addition, we will point out specific aspects of our results that are likely to depend critically on our zero-temperature assumption. After all, it is now understood that for some mechanical properties of networks, the zero temperature presents a singular limit as in the case of rigidity percolation.

Real F-actin networks formed from an actin monomer solution have a complex geometry, arising from the dynamic growth and branching of filaments 1, 9]. For computational efficiency, we ignore such complications and consider static, isotropic networks of monodisperse filaments of length $L$. Each filament is represented by a line segment deposited with random position and orientation to a two-dimensional rectangular shear cell. Intersections are identified as permanent, freely-rotating cross links, to mimic e.g. the attachments of double-headed myosin molecules in an ATP-deficient solution [10. The mean distance between cross links is $l_{\mathrm{c}}$ as measured along a filament. Deposition continues until the desired cross link density $L / l_{\mathrm{c}}$ has been reached.

The network is represented by the set of mobile nodes $\left\{\mathbf{x}_{i}\right\}$ consisting of all cross links and midpoints between cross links (the latter so as to include the dominant bending mode). The total system energy $\mathcal{H}\left(\left\{\mathbf{x}_{i}\right\}\right)$ is then expressed in terms of the $\left\{\mathbf{x}_{i}\right\}$ using a discrete version of (11). Within our linearized scheme, this $\mathcal{H}\left(\left\{\mathbf{x}_{i}\right\}\right)$ is a high-dimensional paraboloid with a unique global minimum, corresponding to the state of mechanical equilibrium at $T=0$. For the initially unstressed network, this minimum corresponds to zero deformation. Depending on whether we wish to measure the shear modulus $G$ or the Young's modulus $Y$, a shear or uniaxial strain $\gamma$ is applied across the periodic boundaries in a LeesEdwards manner. This moves the global minimum to a new, non-trivial position, which we numerically find using the preconditioned conjugate gradient method. The stored energy per unit area can then be calculated, which is $\gamma^{2} / 2$ times $G$ or $Y[8]$ within our linear approximation, and hence the network modulus can be extracted. This procedure is repeated for different network realizations until a reliable estimate of $G$ or $Y$ has been attained. Further simulation details can be found in [11].

Apart from the system size, there are three length scales in the problem: two geometric lengths $L$ and $l_{\mathrm{c}}$, and a third material length scale deriving from the stretching and bending modulii, $l_{\mathrm{b}}=\sqrt{\kappa / \mu}$. On dimensional grounds, the modulii can be written in the form $G=\frac{\mu}{L} f\left(L, l_{\mathrm{c}}, l_{\mathrm{b}}\right)$ (with a similar expression for $Y$ ), provided that the system size is sufficiently large that finite size effects can be ignored, as applies to all of the results presented here.

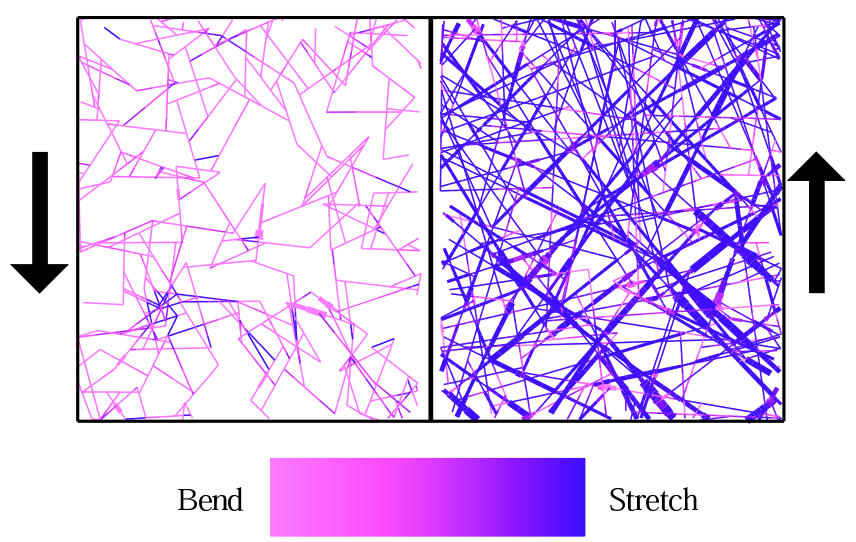

FIG. 1: (Color online) Superimposed examples of the energy distribution throughout networks of cross link densities (left) $L / l_{\mathrm{c}} \approx 8.99$ and (right) $L / l_{\mathrm{c}} \approx 46.77$ with $l_{\mathrm{b}} / L=0.006$ under the given shear strain. The thickness of each segment is proportional to the energy density per unit length, with a minimum so that all filaments are visible, and the calibration bar shows what proportion of the total energy is due to stretching.

Results are given in Fig. 2 for the shear modulus $G$ and the Poisson ration $\nu=2 Y / G-1$. $G$ monotonically increases with the cross link density $L / l_{\mathrm{c}}$ and the ratio $l_{\mathrm{b}} / L$. Both $G$ and $Y$ simultaneously vanish at the rigidity percolation transition $\left[L / l_{c}\right]_{\mathrm{crit}} \approx 5.9$, irrespective of $l_{b}$. This is consistent with the more precise value $\left[L / l_{\mathrm{c}}\right]_{\text {crit }} \approx 5.932$ found by Latva-Kokko et al. [12] using the combinatorial "pebble game" method. We find that $G$ and $Y$ scale near the transition as $\sim\left(L / l_{\mathrm{c}}-\left[L / l_{\mathrm{c}}\right]_{\text {crit }}\right)^{f}$ with $f=3.0 \pm 0.2$, placing it in a distinct universality class from both central-force rigidity and bond bending without free rotation at cross links 13 . The variation of $\nu$ is more subtle if at all, varying from $\approx 0.5$ for high densities to $\approx 0.35 \pm 0.1$ near the critical point (for comparison, standard stability considerations require $-1 \leq \nu \leq 1$ in $2 \mathrm{D})$. Further details of the scaling behavior near the tran- 
sition will be presented elsewhere [11]. We remark that at finite temperatures $G$ will remain non-zero above the conductivity percolation transition at $L / l_{\mathrm{c}} \approx 5.42$, but it is not clear if this small- $G$ behavior is experimentally observable for the macromolecules under consideration here.

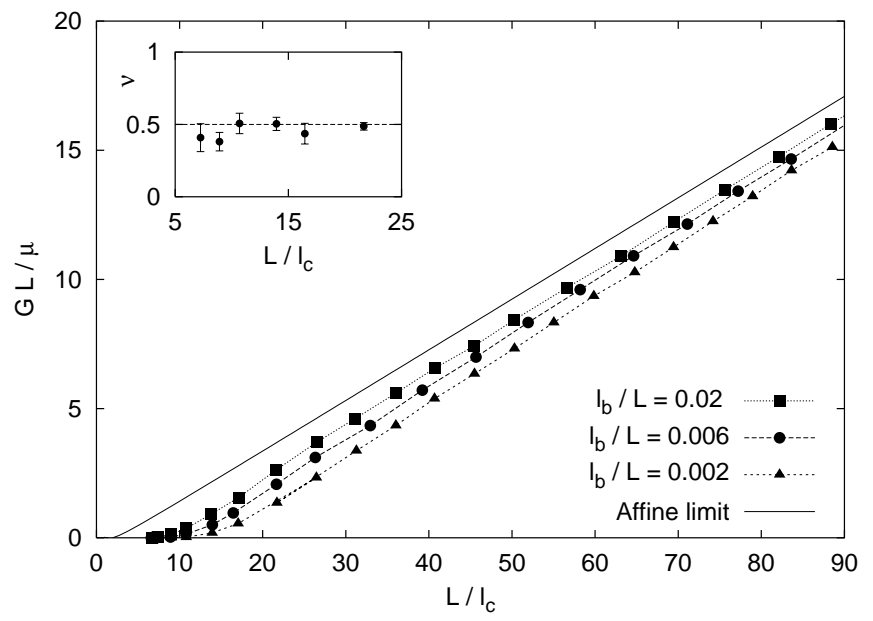

FIG. 2: Dimensionless shear modulus $G L / \mu$ versus the dimensionless cross link density $L / l_{\mathrm{c}}$ for different $l_{\mathrm{b}}$, demonstrating convergence to the affine solution at high densities or molecular weights. The error bars are no larger than the symbols. (Inset) The Poisson ratio $\nu$ for $l_{\mathrm{b}} / L=0.006$.

Away from the critical point, $G$ decreases as the bending modulus $\kappa$ decreases. Indeed, in the limit $\kappa \rightarrow 0$, or equivalently $l_{\mathrm{b}} \rightarrow 0$, all filaments freely bend and the model reduces to a random network of Hookean springs, which is already known to have a vanishing $G$ for all finite cross link densities [14]. In the opposite limit $l_{\mathrm{b}} \rightarrow \infty$, bending along a filament's length becomes prohibitively expensive and response becomes dominated by stretching modes. The same is true of the limit $L / l_{\mathrm{c}} \rightarrow \infty$ with $l_{\mathrm{b}} / L$ fixed, since bending modes of wavelength greater than $l_{\mathrm{c}}$ require an area of network to twist rather than a single filament, and are suppressed. This transition from low $L / l_{\mathrm{c}}$, bending-dominated networks to high $L / l_{\mathrm{c}}$, stretching-dominated ones can be clearly seen Fig. 1

Pure, affine shear, being a combination of rotation and extension, induces only stretching and compression of filaments. Furthermore, networks dominated by stretching modes must be approximately affine. This follows from the observation that, far above the rigidity transition, there can be no orientational disorder without a corresponding cost in bending energy. It is straightforward to derive analytic expressions for the modulii under an affine strain. A rod of length $L$ lying at an angle $\theta$ to the $x$-axis will undergo a relative change in length $\delta L / L=\gamma_{\mathrm{xy}} \sin \theta \cos \theta$ in response to an affine strain field $\gamma_{\mathrm{xy}}$. According to (11) with $\delta \theta=0$, the energy cost (after uniformly averaging over all angles $\theta$ ) is $\delta \mathcal{H}=\mu L \gamma_{\mathrm{xy}}^{2} / 16$. To calculate $G$, we need to express the number of rods per unit area $N$ as a function of $L$ and $l_{\mathrm{c}}$. The exact expression is easy to derive, but for current purposes it is sufficient to use the approximate relation $L / l_{\mathrm{c}} \approx(\alpha-1) /(1-2 / \alpha)$ with $\alpha=2 L^{2} N / \pi$, valid for $L / l_{\mathrm{c}} \approx 5$ and greater (note that the full expression is monotonic). A further correction removes the dangling ends of the rods by renormalizing the rod lengths to $L-2 l_{\mathrm{c}}$. Then using $G=2 \mathcal{H} / \gamma_{\mathrm{xy}}^{2}$,

$$
G_{\text {affine }}=\frac{\pi}{16} \frac{\mu}{L}\left(\frac{L}{l_{\mathrm{c}}}+2 \frac{l_{\mathrm{c}}}{L}-3\right)
$$

so that $G \sim(\pi / 16) \mu / l_{\mathrm{c}}$ as $L / l_{\mathrm{c}} \rightarrow \infty$. Expression (2) is plotted in Fig. 2 and gives a reasonable approximation to the data, with the agreement improving as $L / l_{\mathrm{c}}$ increases. The same calculations can be repeated to give $Y_{\text {affine }}=$ $3 G_{\text {affine }}$ and hence a Poisson ratio $\nu_{\text {affine }}=0.5$, which is also plotted in the figure. Perhaps surprisingly, we find remarkably good agreement between the measured $\nu$ and the affine value, even close to the rigidity transition, where the affine approximation fails.

We observe that there is an apparent crossover from a bending-dominated, non-affine regime for either high molecular weight $(L)$ or for high density. The natural measure for this is the ratio of filament length to average distance between crosslinks. This can be understood by the fact that, unlike networks of flexible polymers, where segments along a single polymer between crosslinks appear to behave as effectively independent network strands, the segments of semiflexible filaments between crosslinks act in series. Thus, segments far from free ends are forced to deform nearly affinely by the many constraints on their neighboring segments imposed by crosslinks. This suggests a physical picture in which non-affine deformations are primarily associated with less constrained free ends. Let $\lambda$ denote the range of such non-affine regions near the filament ends. We expect this length $\lambda\left(l_{c}, l_{b}\right)$ to be a function of the local density of filaments (measured by $l_{c}$, the distance between crosslinks) and the material length $l_{b}$. Then, what determines the degree to which the network is affine or not is the relative size of the affine to non-affine regions, or the ratio $L / \lambda$. We find that, sufficiently far above the transition, $G / G_{\text {affine }}$ collapses to a single master curve with the empirical choice $\lambda=\sqrt[3]{l_{c}^{4} / l_{b}}$, as shown in Fig. B (the origin of this length scale is discussed elsewhere [11]). This confirms the existence of a fundamental length scale $\lambda$ for non-affine deformations along the filament backbone, as well as two physically distinct regimes: non-affine behavior for $L \lesssim \lambda$ and affine behavior for $L \gtrsim \lambda$. In the first of these, we note that the modulus depends only on $\kappa$ and not on $\mu$, signalling a bending dominated regime, as predicted by Kroy and Frey 4, 15. Similar results have been found independently [16]. Note that the crossover can be reached at any fixed density by varying $l_{\mathrm{b}}$ alone, and thus represents distinct physics from the percolation transition, which exists only at a specific density. Hence 
we use absolute density, rather than its value relative to the transition, in our scaling function.

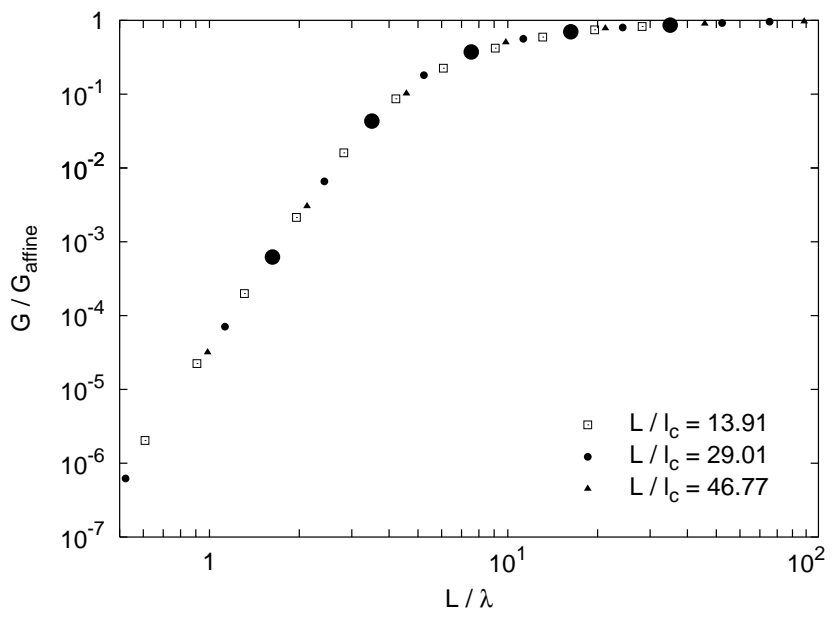

FIG. 3: The master curve of $G / G_{\text {affine }}$ plotted against $L / \lambda$ with $\lambda=\sqrt[3]{l_{\mathrm{c}}^{4} / l_{\mathrm{b}}}$. The enlarged points for $L / l_{\mathrm{c}} \approx 29.09$ correspond to the same parameter values as in Fig. 4

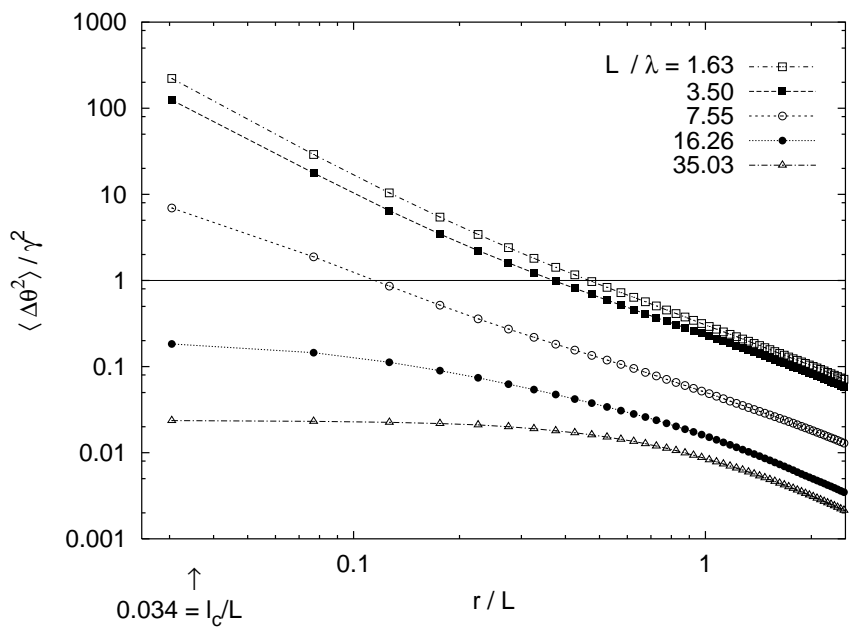

FIG. 4: Plot of the affinity measure $\left\langle\Delta \theta^{2}(r)\right\rangle$ normalised to the magnitude of the imposed strain $\gamma$ against distance $r / L$, for different $l_{\mathrm{b}} / L$. The value of $r$ corresponding to the mean distance between crosslinks $l_{\mathrm{c}}$ is also indicated, as is the solid line $\frac{1}{\gamma^{2}}\left\langle\Delta \theta^{2}(r)\right\rangle=1$, which separates affine from non-affine networks to with an order of magnitude. In all cases, $L / l_{\mathrm{c}} \approx$ 29.1 and the system size was $W=\frac{15}{2} L$.

Our findings demonstrate that the degree of affinity of the deformation field does depend on the length scale on which one looks. This is shown in Fig. 4 where the quantity $\left\langle\Delta \theta^{2}(r)\right\rangle$ is plotted against $r$ for different values of $L / \lambda .\left\langle\Delta \theta^{2}(r)\right\rangle$ is the square deviation of the angle of rotation $\theta$ between two points separated by a distance $r$, relative to the affine equivalent. This monotonically decreases for increasing $r$, suggesting that the deformation appears more affine when viewed on larger length scales. Furthermore, the deviation from affinity at the mesh scale $r=l_{\mathrm{c}}$ is small for networks with $G \approx G_{\text {affine }}$, and large for those with $G \ll G_{\text {affine, }}$, as seen by comparing Figs. 4 and 3 Thus we can reiterate the main findings of our work: (1) there are two qualitatively distinct regimes, one affine and the other non-affine; (2) the physics of the crossover between these is distinct from the rigidity transition; and (3) the crossover is governed by a new length scale $\lambda$, where, e.g., affine behavior is seen for filament lengths a few times this length. Since this length is expected to be of order the distance between crosslinks, real networks can be in either regime depending on the length distribution.

AJL would like to acknowledge the hospitality of the Vrije Universiteit. DAH was partly funded by a European Community Marie Curie Fellowship. This work is supported in part by the National Science Foundation under Grant Nos. DMR98-70785 and PHY99-07949.

[1] B. Alberts, D. Bray, J. Lewis, M. Raff, K. Roberts, and J.D. Watson, Molecular Biology of the Cell (Garland, New York, 1994); E.L. Elson, Annu. Rev. Biophys. Biophys. Chem. 17, 397 (1988); P.A. Janmey, Curr. Opin. Cell Biol. 3, 4 (1991).

[2] M. Rubinstein and R. H. Colby, Polymer Physics (Oxford Univ, London, 2003).

[3] F.C. MacKintosh, J. Käs and P.A. Janmey, Phys. Rev. Lett. 75, 4425 (1995).

[4] K. Kroy and E. Frey, Phys. Rev. Lett. 77, 306 (1996).

[5] R.L. Satcher and C.F. Dewey, Biophysical Journal 71, 109 (1996).

[6] P. G. de Gennes, Scaling Concepts in Polymers Physics (Cornell, Ithaca, 1979); M. Doi and S. F. Edwards, Theory of Polymer Dynamics (Oxford University Press, New York, 1986).

[7] M. Fixman and J. Kovac, J. Chem. Phys. 58, 1564 (1973); C. Bustamante, J. F. Marko, E. D. Siggia, and S. Smith, Science 265, 1599 (1994).

[8] L.D. Landau and E.M. Lifschitz, Theory of Elasticity, 2nd ed. (Oxford, Pergamon Press, 1986).

[9] F.C. MacKintosh and P.A. Janmey, Curr. Opin. Sol. St. and Mat. Sci. 2, 350 (1997).

[10] D. Humphrey, C. Duggan, D. Saha, D. Smith and J. Käs, Nature 416, 413 (2002).

[11] D.A. Head, A.J. Levine and F.C. MacKintosh, in preparation.

[12] M. Latva-Kokko and J. Timonen, Phys. Rev. E 64, 066117 (2001).

[13] M. Sahimi, Phys. Rep. 306, 213 (1998); M. Plischke and B. Joós, Phys. Rev. Lett. 80, 4907 (1998).

[14] M. Kellomäki, J. Åström and J. Timonen, Phys. Rev. Lett. 77, 2730 (1996).

[15] E. Frey, K. Kroy, J. Wilhelm, in The Wiley Polymer Networks Group Review, Volume 2, Synthetic versus Biological Networks B. T. Stokke and A. Elgsaeter, Eds (WileyEurope, 2000).

[16] J. Wilhelm and E. Frey, submitted to Phys. Rev. Lett. 